\title{
Contriving of Orthogonal Frequency Division Multiplexing (OFDM) Transmitter with the Aid of Phase Shift Keying (PSK) and a 256 bit Grayscale Image
}

\author{
Sudipta Ghosh \\ Student, SECE \\ Lovely Professional University \\ Jalandhar, INDIA
}

\author{
Chandika Mohan Babu \\ Asst. Professor, SECE \\ Lovely Professional University \\ Jalandhar, INDIA
}

\begin{abstract}
Orthogonal frequency division multiplexing (OFDM) is a special case of multicarrier transmission, where a single data stream is transmitted over a number of lower rate subcarriers. Orthogonal frequency division multiplexing (OFDM) has been chosen as modulation technique for different application wireless communications. OFDM can provide large data rates with sufficient robustness to radio channel impairments. The purpose of this paper is to provide a MATLAB simulation of the basic processing involved in the generation and reception of an OFDM signal in a physical channel and to provide a description of each of the steps involved. For this purpose, we shall use a 256 bit gray scale image as the input/data to be transmitted.
\end{abstract}

\section{General Terms}

Fast fourier transform (FFT), Inverse fourier Transform (IFFT), Pulse Shaping, Filters

\section{Keywords}

Orthogonal Frequency Division Multiplexing (OFDM), Gray scale image

\section{INTRODUCTION}

In telecommunication, a communication system is a collection of individual communication networks, transmission systems, relay stations, tributary stations, and data terminal equipment usually capable of interconnection and inter operation to form an integrated whole. The components of a communication system serve a common purpose, are technically compatible, use common procedures, respond to controls, and operate in unison. Telecommunications is a method of communication (e.g. for sports broadcasting, mass, media, etc.). A communication sub system is a functional unit or operational assembly that is smaller than the larger assembly under consideration. The principle of multi-carrier transmission is to convert a serial high rate data stream on to multiple parallel low rate sub-streams. Each sub-stream is modulated on another sub-carrier. Since the symbol rate on each subcarrier is much less than the initial serial data symbol rate, the effects of delay spread, i.e. ISI, significantly decrease, reducing the complexity of the equalizer. OFDM is a low complex technique used to modulate multiple sub-carriers efficiently by using digital signal processing [9]. Note that the three-dimensional time/frequency/power density representation is used to illustrate the principle of various multi-carrier and multi-carrier spread spectrum systems. A cuboid indicates the three-dimensional time/frequency/power density range of the signal, in which most of the signal energy is located and does not make any statement about the pulse or spectrum shaping. As such it has received much attention and has been proposed for many other applications, including local area networks and personal communication systems. OFDM is a type of multichannel modulation that divides a given channel into many parallel sub channels or subcarriers, so that multiple symbols are sent in parallel. The type of OFDM that we will describe in this article uses the discrete Fourier transform (DFT) with a cyclic prefix. DFT (implemented with a fast Fourier transform (FFT)) and the cyclic prefix have made OFDM both practical and attractive to the radio link designer. A similar multichannel modulation scheme, discrete multi tone (DMT) modulation, has been developed for static channels such as the digital subscriber loop. DMT also uses DFTs and the cyclic prefix but has the additional feature of bit-loading which is generally not used in OFDM.

OFDM also has some drawbacks. Because OFDM divides a given spectral allotment into many narrow subcarriers each with inherently small carrier spacing, it is sensitive to carrier frequency errors. Furthermore, to preserve the orthogonality between subcarriers, the amplifiers need to be linear. OFDM systems also have a high peak-to-average power ratio or crest-factor, which may require a large amplifier power backoff and a large number of bits in the analog-to-digital (A/D) and digital-to-analog (D/A) designs. All these requirements can put a high demand on the transmitter and receiver design [10].

\section{ORTHOGONAL FREQUENCY DIVISION MULTIPLEXING (OFDM)}

OFDM is a multi-carrier modulation technique where data symbols modulate a sub-carrier which is taken from orthogonally separated sub-carriers with a separation of ' $\mathrm{fk}$ ' within each sub-carrier. Here, the spectra of sub-carrier are overlapping; but the sub-carrier signals are mutually orthogonal, which is utilizing the bandwidth very efficiently. To maintain the orthogonality, the minimum separation between the sub-carriers should be ' $f_{\mathrm{K}}$ ' to avoid ICI (Inter Carrier Interference).By choosing the sub-carrier spacing properly in relation to the channel coherence bandwidth. OFDM can be used to convert a frequency selective channel into a parallel collection of frequency flat sub-channels. Techniques that are appropriate for flat fading channels can 
then be applied in a straight forward fashion.

\section{SIGNAL MODEL}

A communication system with multi-carrier modulation transmits $\mathrm{N}_{\mathrm{C}}$ complex-valued source symbol $\mathrm{S}_{\mathrm{N}}, \mathrm{N}=0$, $\ldots, N_{C}-1$, in parallel on $\mathrm{N}_{\mathrm{C}}$ sub-carriers. The source symbols may, for instance, be obtained after source and channel coding, interleaving, and symbol mapping [2]. The source symbol duration $\mathrm{T}_{\mathrm{S}}$ of the serial data symbols results after serial- to-parallel conversion in the OFDM symbol duration.

$$
T_{s}=N_{c} T_{d}
$$

The principle of OFDM is to modulate the $\mathrm{N}_{\mathrm{C}}$ sub-streams on sub-carriers with a spacing of

$$
F_{s}=1 / T_{s}
$$

in order to achieve orthogonality between the signals on the Nc sub-carriers, presuming a rectangular pulse shaping. The $\mathrm{Nc}$ parallel modulated source symbols $\mathrm{Sn}, \mathrm{n}=0, \ldots, \mathrm{N}_{\mathrm{C}}-1$, are referred to as an OFDM symbol [8]. The complex envelope of an OFDM symbol with rectangular pulse shaping has the form

$$
x(t)=\frac{1}{N_{c}} \sum_{n=0}^{N_{c}-1} S_{n} e^{j 2 \pi f_{n} t} \quad, 0 \leq t<T_{s}
$$

The Nc sub-carrier frequencies are located at

$$
f_{n}=\frac{n}{T_{s}}, n=0, \ldots \ldots, N_{c}-1
$$

The symbols $\mathrm{S}_{\mathrm{n}}, \mathrm{n}=0, \ldots, \mathrm{N}_{\mathrm{C}}-1$, are transmitted with equal power. The dotted curve illustrates the power density spectrum of the first modulated sub-carrier and indicates the construction of the overall power density spectrum as the sum of $\mathrm{N}_{\mathrm{C}}$ individual power density spectra, each shifted by $\mathrm{F}_{\mathrm{S}}$. For large values of $\mathrm{N}_{\mathrm{C}}$, the power density spectrum becomes flatter in the normalized frequency range of $-0.5_{-}$fTd -0.5 containing the $\mathrm{N}_{\mathrm{C}}$ subchannels. Only sub-channels near the band edges contribute to the out-of-band power emission. Therefore, as $\mathrm{N}_{\mathrm{C}}$ becomes large, the power density spectrum approaches that of single carrier modulation with ideal Nyquist filtering [1]. A key advantage of using OFDM is that multi-carrier modulation can be implemented in the discrete domain by using an IDFT, or a more computationally efficient IFFT. When sampling the complex envelope $x(t)$ of an OFDM symbol with rate $1 / \mathrm{Td}$ the samples are

$$
x_{v}=\frac{1}{N_{c}} \sum_{0}^{N_{c}-1} S_{n} e^{j 2 \pi n v / N_{c}}, v=0, \ldots \ldots, N_{c}-1
$$

The normalized power spectrum of OFDM is shown in Fig. 1

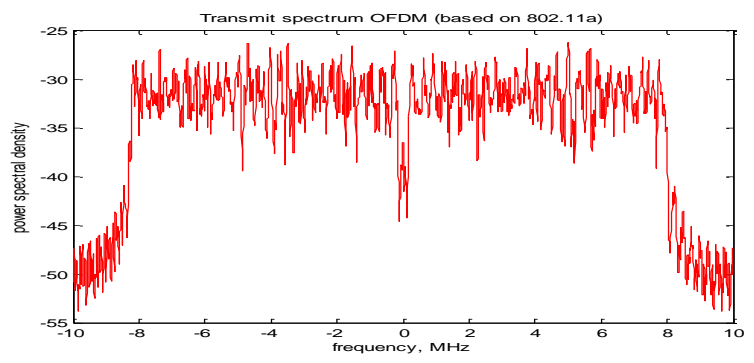

Figure 1. Normalized power spectrum of an OFDM signal

\section{Phase Shift Keying (PSK)}

Phase-shift keying (PSK) is a digital modulation scheme that conveys data by changing, or modulating, the phase of a reference signal (the carrier wave). Any digital modulation scheme uses a finite number of distinct signals to represent digital data. PSK uses a finite number of phases; each assigned a unique pattern of binary digits. Usually, each phase encodes an equal number of bits. Each pattern of bits forms the symbol that is represented by the particular phase. The demodulator, which is designed specifically for the symbol-set used by the modulator, determines the phase of the received signal and maps it back to the symbol it represents, thus recovering the original data. This requires the receiver to be able to compare the phase of the received signal to a reference signal such a system is termed coherent (and referred to as CPSK). Alternatively, instead of operating with respect to a constant reference wave, the broadcast can operate with respect to itself. Changes in phase of a single broadcast waveform can be considered the significant items. In this system, the demodulator determines the changes in the phase of the received signal rather than the phase (relative to a reference wave) itself. Since this scheme depends on the difference between successive phases, it is termed differential phase-shift keying (DPSK). DPSK can be significantly simpler to implement than ordinary PSK since there is no need for the demodulator to have a copy of the reference signal to determine the exact phase of the received signal (it is a non-coherent scheme). In exchange, it produces more erroneous demodulations [1].

The simplest form of PSK is binary phase-shift keying (BPSK), where $\mathrm{N}=1$ and $\mathrm{M}=2$. Therefore, with BPSK, two phases $\left(2^{1}=2\right)$ are possible for the carrier. One phase represents logic 1 , and the other phase represents logic 0 . As the input digital signal changes state (i.e., from 1 to 0 or from a 0 to a 1), the phase of the output carrier shifts between two angles that are separated by $180^{\circ}$. Hence, other names for BPSK are phase reversal keying (PRK) and bi phase modulation. BPSK is a form of square-wave modulation of a continuous wave (CW) signal [3]. A binary phaseshift keying (BPSK) signal can be defined by

$$
s(t)=A m(t) \cos \left(2 \pi f_{c} t\right), 0 \leq t \leq T
$$

where $\mathrm{A}$ is a constant, $\mathrm{m}(\mathrm{t})=+1$ or $-1, \mathrm{fc}$ is the carrier frequency, and $\mathrm{T}$ is the bit duration. The signal has a power $\mathrm{P}$ $=A 2 / 2$, so that $A=2 P$. Thus equation 6 can be written as

$$
s(t)= \pm \sqrt{2 P} \cos \left(2 \pi f_{c} t\right)
$$




$$
\begin{aligned}
& = \pm \sqrt{P T} \sqrt{\frac{2}{T}} \cos \left(2 \pi f_{c} t\right) \\
& = \pm \sqrt{E} \sqrt{\frac{2}{T}} \cos \left(2 \pi f_{c} t\right)
\end{aligned}
$$

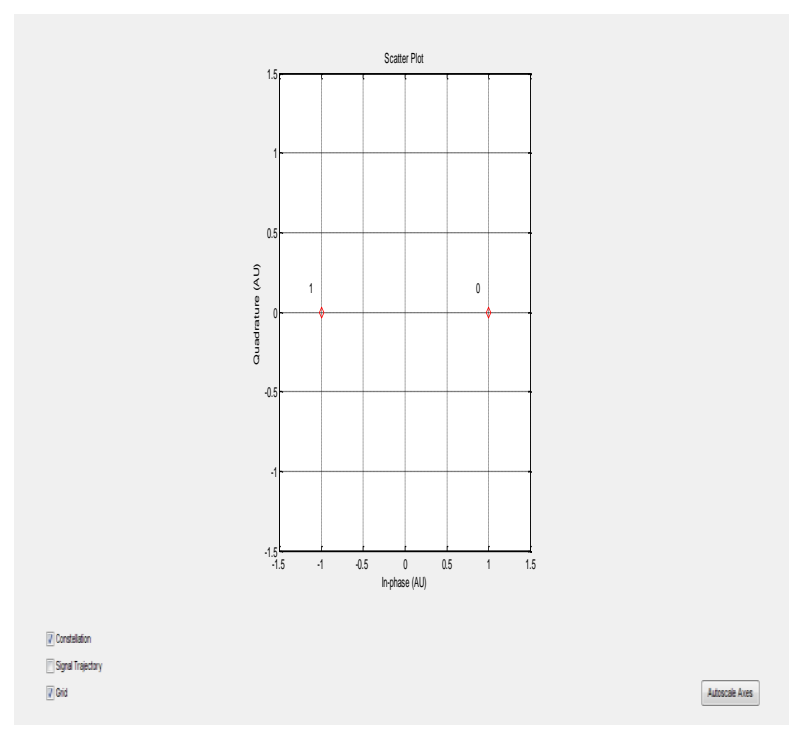

Figure 2. Constellation diagram of BPSK

Figure 3 shows the BPSK signal sequence generated by the binary sequence, the modulating signal and the modulated BPSK signal for the input sequence 00011011

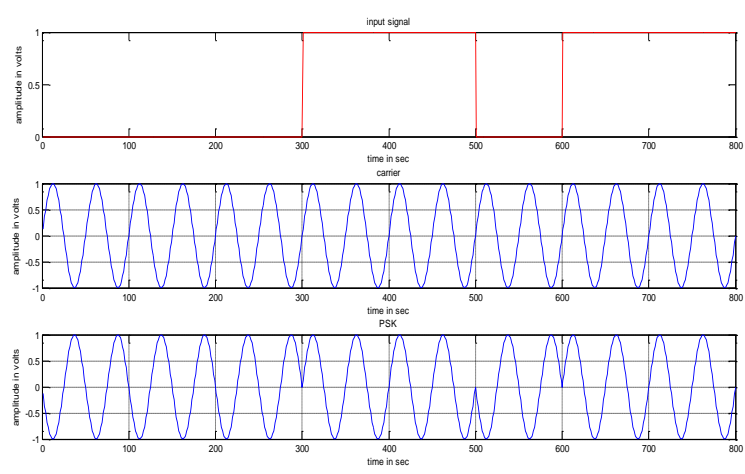

Figure 3. BPSK modulated signal

\section{Transmitter Model using MATLAB}

The core of the OFDM transmitter is the modulator, which modulates the input data stream frame by frame. Data is divided into frames based on the variable symbol per frame, which refers to the number of symbols per frame per carrier. It is defined by symbol per frame $=\operatorname{ceil}\left(2^{\wedge} 13 /\right.$ carrier count $)$. This limits the total number of symbols per frame (symbol per frame $*$ carrier count) within the interval of $\left[2^{\wedge} 13\right.$, $\left.2^{*}\left(2^{\wedge} 13-1\right)\right]$, or $[8192,16382]$. However, the number of carriers typically would not be much greater than 1000 in this simulation, thus the total number of symbols per frame would typically be under 10,000 . This is an experimentally reasonable number of symbols that one frame should keep under for this MATLAB program to run efficiently; thereby symbol per frameis defined by the equation shown above. If the total number of symbols in a data stream to be transmitted is less than the total number of symbols per frame, the data would not be divided into frames and would be modulated all at once. Even if the data stream is not sufficiently long enough to be divided into multiple frames, two frame guards with all zero values and in a length of one symbol period are still added to both ends of the modulated time signal. This is to assist the receiver to locate the beginning of the substantial portion of the time signal. For modulated signals with multiple frames, a frame guard is inserted in between any two adjacent frames as well as both ends of the cascaded time signal. Finally, a pair of headers is padded to both ends of the guarded series of frames. The headers are scaled to the RMS level of the modulated time signal. Figure 4 shows the 256 bit gray scale input image. Using the above methodologies and formulas a transmitter system was developed in MATLAB. The simulation plots obtained are shown in figures 5 to 8 .

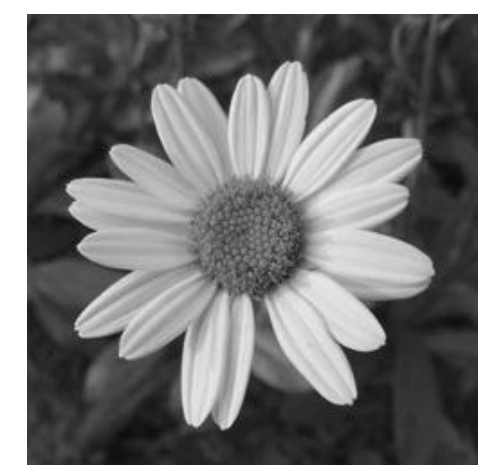

Figure 4. Input 256 bit grayscale image

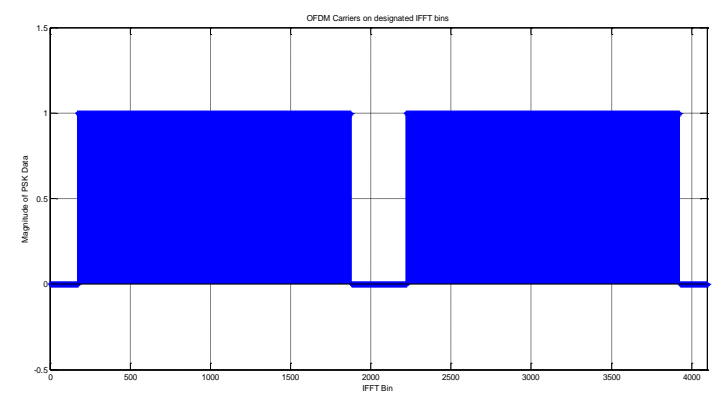

Figure 5. OFDM carriers

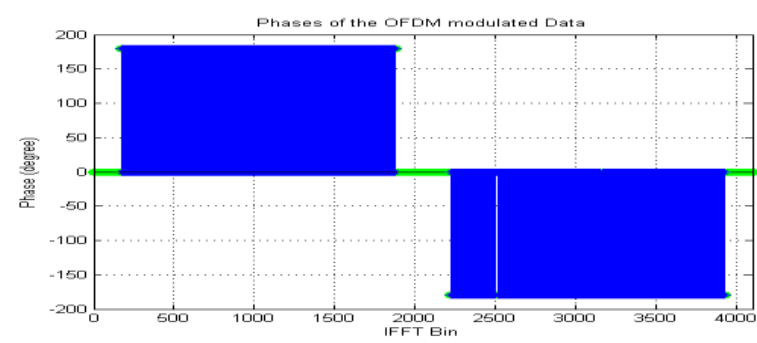

Figure 6. Phase of the OFDM modulated data 


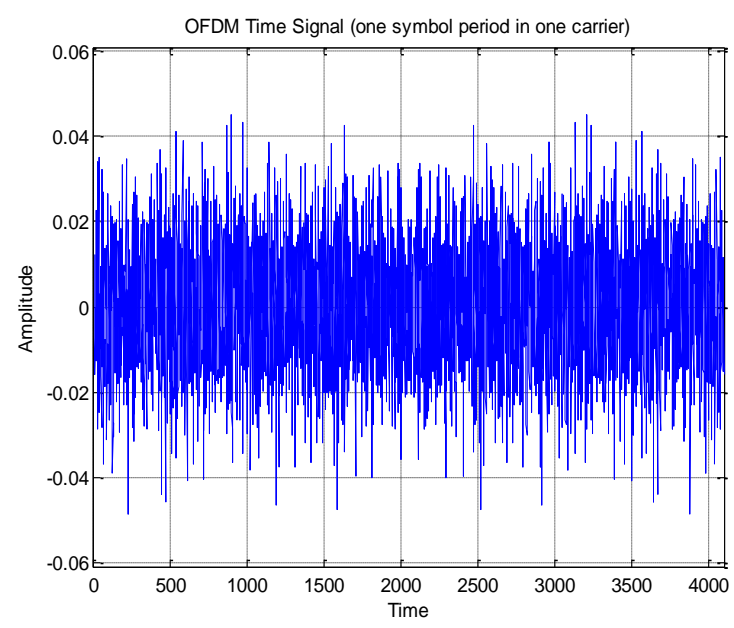

Figure 7. OFDM time signal

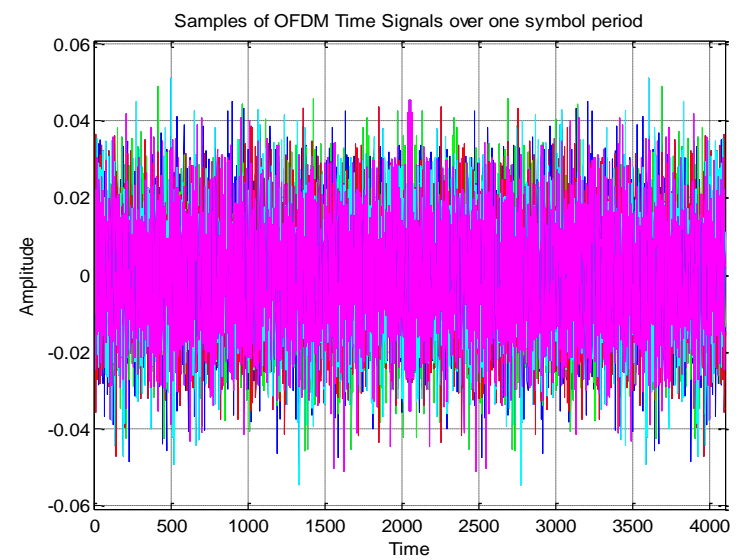

Figure 8. Samples of OFDM time signals over one symbol period

We have used AWGN noise in order to simulate a noisy channel interface. Figure 10 shows a screen shot of the data that was used as input and the various data obtained during the simulation. In this simulation we used the length of the IIFT as 4096 and also used 1705 sub carriers which is very much similar to that used in DVB-T, another application od OFDM.

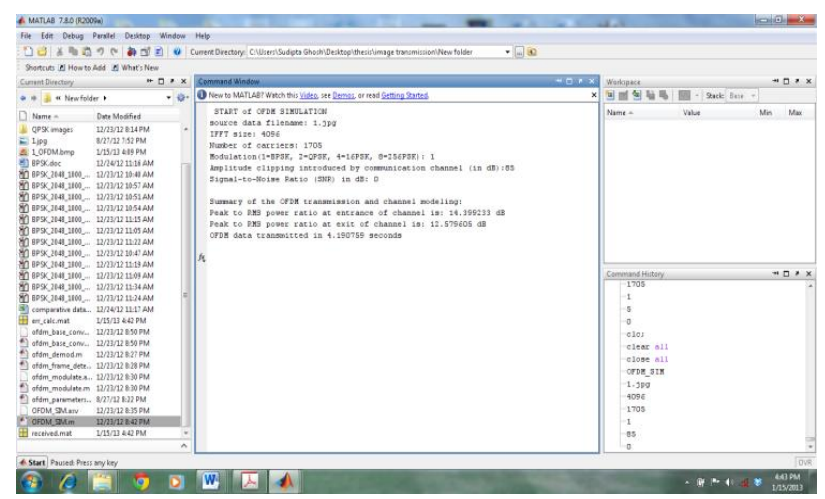

\section{CONCLUION}

In this we have discussed only the transmitter model have been discussed in details along with their spectral plots. Work is being carried on in order to design a receiver that is compatible with this transmitter model and gives us a more detailed result including its BER (bit error rate) for varied rates of signal to noise ratio (SNR). This whole paper is based on the simulation results obtained from the simulation of the discussed model in MATLAB. Further studies are being done in order to enhance the system by implementing corrective measures like FEC coding and so on.

\section{ACKNOWLEDGEMENT}

We would like to thank Mr. Chandika Mohan Babu, my guide, and Mr. Ankit Bass for being a constant source of inspiration and support while writing this research paper. Without their help this would not have been a success.

\section{REFERENCES}

[1] David M Beams, "Electrical Measurement, Signal Processing, and Displays", CRC Press.

[2] C. Yuen, Y. Wu, and S. Sun, "Comparative study of open-loop transmit diversity schemes for four transmit antennas in coded OFDM systems," In Proceeding of IEEE conference on Vehicular Technology, pp. 482-485, (Baltimore, MD)September 2004

[3] A. Boariu and D.M. Ionescu "A class of nonorthogonal rate-one space time block codes with controlled interference," IEEE Transactions on Wireless Communications, Vol. 2, Issue 2, pp. 270-395, March 2003

[4] V. Tarokh, H. Jafarkhani and A. R. Calderbank, "Spacetime block codes from orthogonal designs", IEEE Transactions on Information Theory, Vol. 45, pp. 14561467, July 1999

[5] J. Kim and I. Lee, "Space-time coded OFDM systems with four transmit antennas," In Proceeding of IEEE conference on Vehicular Technology, vol. 2, pp. 24342438, September 2004.

[6] Orthogonal frequency division multiplexing for highspeed optical transmission, by Ivan B. Djordjevic et al

[7] A Novel Construction Technique For Designing Of Video Application Using Wirless 4G, by M.Suman et al.

[8] Engels M. (ed.), "Wireless OFDM Systems: How to Make Them Work" , Boston: Kluwer Academic Publishers.

[9] OFDM Simulation Using Matlab, http://www.ece.gatech.edu/research/labs/sarl/tutorials/OF DM/Tutorial_web.pdf.

[10] Sudipta Ghosh, Ankit Bass, "Implementation of digital video broadcasting terrestrial (DVB-T) using orthogonal frequency division multiplexing (OFDM) on physical media dependent sub layer", IJCA (International Journal of Computer Application), Volume 44, number 22,April 2012, Pg 20 to 25

Figure 9. Screenshot of the simulated data 